\title{
Spatially resolved differential resistance of bulk superconductors by laser-induced heating
}

Robert McLaren and Norman J. Dovichi

Department of Chemistry. University of Alberta. Edmonton. Alberta. T6G $2 G 2$ Canada

Spatially resolved measurements of the change in resistance with temperature of bulk high-temperature superconductors are generated by heating a localized region of a sample with a modulated laser beam. The amplitude of the thermally induced resistance change is related to the heat capacity and derivative of resistance with temperature whereas the phase of the signal is related to the thermal diffusivity of the sample. By moving the sample with respect to the focused laser beam, spatially resolved measurements may be made. Images with $\sim 50-\mu \mathrm{m}$ resolution have been used to locate defects in polycrystalline $\mathrm{Bi}-\mathrm{Sr}-\mathrm{Ca}-\mathrm{Cu}-\mathrm{O}$ sample and to estimate the thermal diffusivity of the bulk material.

The change in resistance with temperature, $d R / d T$, of superconductors is important in the identification of transitions to the superconducting state; sharp decreases in the resistance of the sample occur at the transition temperature. For polycrystalline materials, the sample does not always demonstrate zero resistance below a transition temperature because of the presence of impurities or multiple superconducting phases within the sample. For these polycrystalline samples, the resistance itself is not of primary interest. Instead, $d R / d T$ is of more interest because those regions with large $d R / d T$ are likely undergoing a transition to the superconducting state. Of course, a large $d R$ / $d T$ is not proof of superconductivity. However, measurements of the Meissner effect are associated with the bulk specimen and do not give any information on the spatial distribution of superconducting phases within the sample.

This communication presents, spatially resolved measurement of $d R / d T$ for bulk high-temperature superconductors. Low-temperature scanning electron microscopy has been used to image the physical properties of lowtemperature superconducting tunnel junctions and microbridges.' The same technique has been used to show the limitation of critical current densities by individual grain boundaries in $\mathrm{YBa}_{2} \mathrm{Cu}_{3} \mathrm{O}_{7}$ thin films. ${ }^{2}$ A photothermal technique has also been used to measure the directional anisotropies in the thermal diffusivity of single-crystal $\mathrm{Bi}_{2} \mathrm{Sr}_{2} \mathrm{CaCu}_{2} \mathrm{O}_{8}$ and to image these anisotropies in polycrystalline materials. ${ }^{3}$

The resistance of the sample is measured with a fourprobe ac technique at frequency $f_{i}$ (Fig. 1). To generate the thermally modulated signal, a helium-cadmium laser beam is mechanically chopped at frequency $f_{m}$ and focused on the sample surface between the voltage probes. The amplitude of the thermally modulated signal, related to $d R / d T$, is measured by synchronously detecting the moduiation in the voltage across the sample with a two-phase lock-in amplifier referenced to the upper sideband frequency $\left(f_{m}+f_{c}\right)$. For spatially resolved measurements, the lens is moved in the $x-y$ plane while the sample is held stationary. An alternate signal detection scheme for low modulation frequency uses two lock-in amplifiers in series. The first lock-in is operated with a short time constant and measures the resistance of the sample at $f_{c}$. The second lock-in detects the resistive modulation amplitude at $f_{m}$. Three signals-resistance, modulation amplitude, and modulation phase - can be recorded simultaneously. In the data presented below, the sample is $7 \mathrm{~mm}$ long, $2 \mathrm{~mm}$ wide, and $1 \mathrm{~mm}$ thick and held in contact with a copper cold finger with high thermal conductivity grease.

This laser-induced thermal modulation technique is similar to a very sensitive thermo-optical method for small volume absorbance detection in aqueous solutions. ${ }^{4} \mathrm{~A}$ sim. ple description of the magnitude of the modulation amplitude in solids can be given in the approximation of a homogeneous sample, homogeneous current density, fast optical modulation, and thick sample. The measured voltage $V_{\mathrm{rms}}$ is approximated by

$$
V_{\mathrm{rms}} \cong \frac{I d R}{v} \frac{P(1-r)}{d T} \frac{1}{\rho C_{p} f_{m}}
$$

where $I$ is the current through the sample with volume $v$ between the voltage leads, $P$ is the laser power, $r$ is the reflectance of the surface, $\rho$ is the density of the material, and $C_{p}$ is the sample's heat capacity. The dynamics of heat flow and the thermal conductivity of the sample become more important as the modulation frequency and sample thickness are decreased. A more detailed description of the thermal dynamics induced by an electron beam source in thin films has been presented. ${ }^{3}$ The modulation amplitude is proportional to $d R / d T$ and inversely proportional to sample volume, density, and heat capacity. To obtain good signal-to-noise ratio for bulk samples, it is necessary to use a low modulation frequency. The spatial resolution, which degrades at low modulation frequency, can be characterized by the dynamic thermal healing length $\eta_{\omega}-\left(D / \pi f_{m}\right)^{1 / 2}$, where $D=k / \rho C_{\rho}$ is the thermal diffusivity of the material with thermal conductivity $k_{.}{ }^{2}$

A two lock-in scheme was used to measure the thermally modulated amplitude and the resistance of a bulk polycrystalline sample of $\mathrm{YBa}_{2} \mathrm{Cu}_{3} \mathrm{O}_{7}$. The thermally modulated amplitude signal maximized at a slightly lower temperature than the resistive transition midpoint, presumably as a result of the local steady-state $(\mathrm{dc})$ temperature rise 


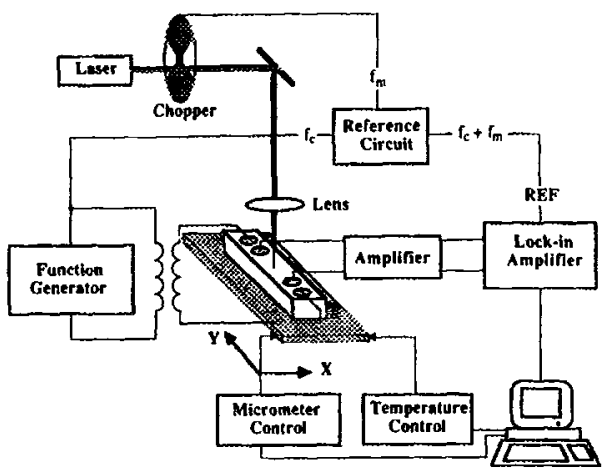

FIG. I. Experimental setup using sideband detection. $f_{\mathrm{c}}$ and $f_{\mathrm{m}}$ are the frequencies used to measure the resistance and thermally-modulated resistance, respectively.

induced by the laser. This temperature shift was approximately $-1 \mathrm{~K}$ for the $13.5-\mathrm{mW}$ laser and increased with laser power. Above the transition temperature, the modulated amplitude slowly fell to a roughly constant level, reflecting the metallic-like behavior of $d R / d T$ in the normal state of the material. Below the transition temperature, the resistance of the sample showed both undetectable resistance and thermally modulated amplitude at $85 \mathrm{~K}$. The zero thermally modulated amplitude signal at zero resistance demonstrates the absence of a significant nonbolometric response of the material to the $442-\mathrm{nm}$ radiation. Nonbolometric responses such as quasiparticle formation allow fast infrared detection. ${ }^{6.7}$ Previously, the absence of a nonbolometric response of $\mathrm{YBa}_{2} \mathrm{Cu}_{3} \mathrm{O}_{7}$ thin films to $632.8-\mathrm{nm}$ and $1.06-\mu \mathrm{m}$ radiation has been reported. ${ }^{8}$

Figure 2 presents the thermal modulation amplitude [Fig. 2(a)] and phase [Fig. 2(b)], numerically differentiated bulk resistance [Fig. 2(c)], and the bulk resistance [Fig. 2(d)] of a sample of nominal composition $\mathrm{Bi}_{4} \mathrm{Sr}_{3} \mathrm{Ca}_{3} \mathrm{Cu}_{6} \mathrm{O}_{18+x}$. The laser was loosely focused in the 5 $\mathrm{mm}$ region between the voltage leads. Three transitions are observed in the thermally modulated amplitude signal: a large, broad transition centered at $72 \mathrm{~K}$ and two minor transitions at 105 and $116 \mathrm{~K}$ [Fig. 2(a), inset]. Multiple structural superconducting phases in the $\mathrm{Bi}-\mathrm{Sr}-\mathrm{Ca}-\mathrm{Cu}-\mathrm{O}$ system are well known and can be represented by the general formula $\mathrm{Bi}_{2} \mathrm{Sr}_{2} \mathrm{Ca}_{n-1} \mathrm{Cu}_{n} \mathrm{O}_{2 n+4}$, where $n$ is the number of adjacent $\mathrm{Cu}-\mathrm{O}$ layers. ${ }^{9} 10$ The transition temperatures increases with $n$ and have been reported at $T_{c}=60 \pm 20,82 \pm 8$, and $107 \pm 3 \mathrm{~K}$ for the $n=1,2,3$ members of the series, respectively. ${ }^{10}$ In Fig. $2(a)$, the 72-K transition is very broad and may not be due to a pure phase. The onset of this transition at $\sim 90 \mathrm{~K}$ may be due to the presence of the 2212 phase. The slow drop in resistance below this temperature, Fig. 2(d), is characteristic of the Bi-Sr-Ca-Cu-O system. Zero resistance is not achieved above $70 \mathrm{~K}$ for this sample, the lowest temperature reached with our refrigeration system. Two minor transitions are shown in the inset in Fig. 2(a). The transition centered at $105 \mathrm{~K}$ is assigned to the presence of the 2223 phase. The transition at $116 \mathrm{~K}$ may be due to a higher-order phase, $n>3$, and may be responsible for reports of superconductivity onset near $120 \mathrm{~K}$ in this system by magnetic susceptibility measurements."

The numerically differentiated resistance data in Fig. 2(c) qualitatively match the thermally modulated data in Fig. 2(a). However, the relative intensity of the two lowest
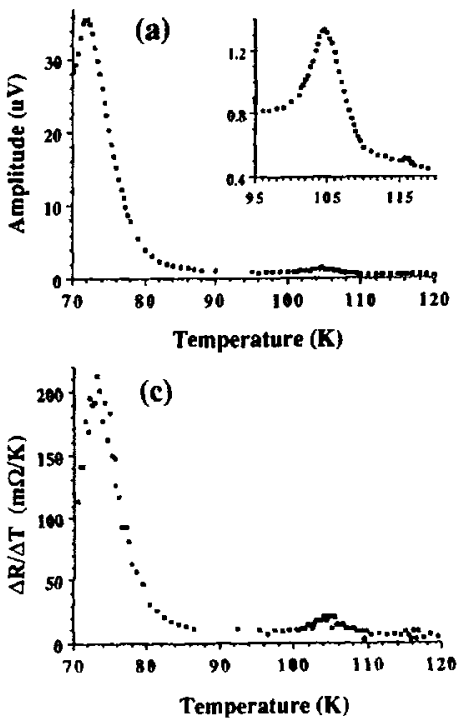
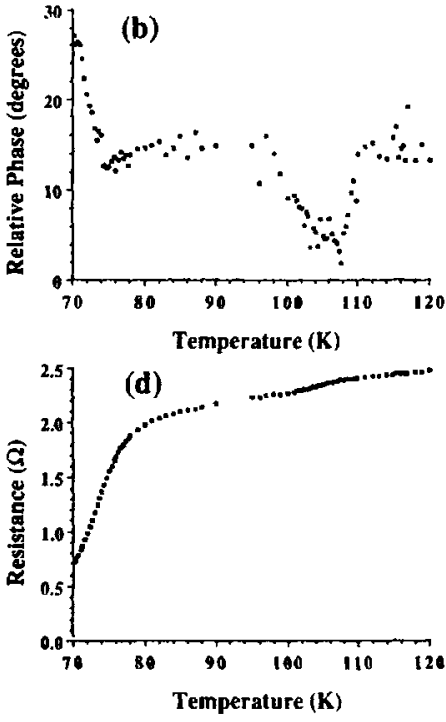

FIG. 2. Temperature-dependent data recorded simultaneously with a two-iock-in detection scheme for a multiphase polycrystalline $\mathrm{Bi}-\mathrm{S} r$ $\mathrm{Ca}-\mathrm{Cu}-\mathrm{O}$ sample. (a) Laser-induced thermal modulation amplitude for a modulation frequency of $10 \mathrm{~Hz}$ and $I=7.3 \mathrm{~mA}$. (b) Relative phase of the laser-induced thermal modulation amplitude signal. (c) Numerical derivative of the resistance data. (d) Resistance. 
(a)

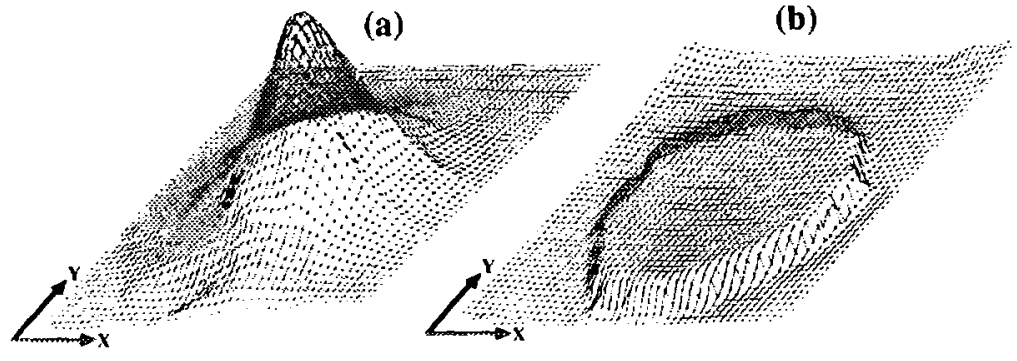

FIG. 3. Thermal modulation images recorded simultaneously of the same Bi-Sr-Ca-Cu-O sample from Fig. 2 for a modulation frequency of $50 \mathrm{~Hz}$ at $72 \mathrm{~K}$. The image is of a $1 \times 1 \mathrm{~mm}$ area between the voltage leads using $20-\mu \mathrm{m}$ pixels. The electrodes are centered on the $Y$ axis of the figure and located about $2 \mathrm{~mm}$ beyond the efge of the figure. (a) Amplitude. (b) Phase. transitions in the thermally modulated data $\left(H_{72 \mathrm{~K}} / H_{10 \mathrm{~K}}\right)$ is greater than the corresponding ratio in the numerically differentiated data and probably is a result of a gradual decrease in the heat capacity of the material from 105 to $75 \mathrm{~K}$. A much higher signal-to-noise ratio is produced by the thermally modulated technique compared with the numerically differentiated data, in part due to the long time constant an the second lock-in amplifier used to measure the thermally modulated signal and in part due to the inherently noisy process of numerical differentiation.

The relative phase of the laser-induced signal in Fig. 2 (b) is related to the thermal diffusivity of the sample. The exact functional relationship between phase and thermal diffusivity depends on the particular geometry of the sample and is not considered here. However, changes in the diffusivity of the sample at the transition temperature of the sample presumably lead to the sharp changes in the relative phase observed in Fig. 2(b).

Figure 3 presents the thermally modulated amplitude (a) and phase (b) images of the same Bi-Sr-Ca-Cu-O sample as Fig. 2. The images were collected at $72 \mathrm{~K}$ with the double-sideband instrument over a $1 \times 1 \mathrm{~mm}$ area between the voltage leads, $f_{n}=50 \mathrm{~Hz}$. The current path was parallel to the $y$ axis. The thermally modulated signal appears to originate from a point on the current path. Visual inspection of the sample revealed a crack with partially melted material that ran diagonally across the full width of the sample. The large signal is centered at the intersection of the defeet and the current path. The bulk resistance messurement is representative of this region of high resistance whereas the thermally modulated images in both modes can locate the defect. The phase of the thermally modulated signal outlines a central region with different thermal diffusivity than the surrounding sample.

Images collected at higher modulation frequencies, up to $400 \mathrm{~Hz}$. presented a decrease in the apparent width of the defect (at the expense of $S / N$ ) due to decreased dy- namic thermal healing length. The observed variance of the peak is related to the sum of the variances of other experimental parameters, $\sigma_{\text {app }}^{2}=\sigma_{\text {nat }}^{3}+\sigma_{n}^{2}+\sigma_{L}^{2}$, where the variances in the sum are due to the natural defect size- $\sigma_{\text {nat }}$, the thermal healing length $-a_{\eta}$, and the finite spot size of the laser beam- $-\sigma_{L}$. The last contribution to the size of the peak is small for a tightly focused beam and, along with the natural defect size, independent of frequency. A plot of $\sigma_{a p p}^{2}$ vs $1 / f_{m}$ was used to estimate the thermal diffusivity of the material, $D=3.9(2) \mathrm{mm}^{2} \mathrm{~s}$ ' 1 at $72 \mathrm{~K}$. At the highest modulation frequency, the dynamic thermal healing length was about $60 \mu \mathrm{m}$, sufficiently small to ensure that there was little heat flow from the illuminated sample to the cold finger below the 1 -mm-thick sample.

The authors to thank W. Weimer of China Lake Navel Weapons Research Center for helpful discussions and for the donation of a $\mathrm{YBa}_{2} \mathrm{Cu}_{3} \mathrm{O}$, sample. This work was funded by the Natural Sciences and Engineering Research Council of Canada. R. M. acknowledges support by a Province of Alberta Graduate Research Fellowship.

'R. P. Huebener, in Adtances in Electromics and Eltctron Physics, edited by P. W. Hawkes (Academic. New York. 1988). Vol. 70. p.1.

2J. Mannhart, R. Gross. K. Hipler, R. P. Heubener. C. C. Tsuei, D. Dimos, and P. Chaudhari. Science 245. 839 (1989).

J. T. Fanton, D. B. Mitzi, A. Kapitulnik, B. T. Khuri-Yakuh, G. S Kino, D. Gazit, and R. S. Feigelson, Appl. Phys. Let1. 55, 598 ( 1984 ) ${ }^{4} R$. McLaren and N. J. Dovichi, Can. J. Chem. 67, 1178 (1989).

"J R. Clem and R. P. Hutbener, Appl. Phys 51. 2764 (1980)

"Y. Enomoto and T. Murakami, J. Appl. Phys. 59. 3807 (1986).

${ }^{7}$ A. Frenkel, M. A. Saifi, T. Vonkatesan, C. Lin, X. D. Wu, and A. Inam. Appl. Phys. Lell. 54. 1594 (1989).

"W. S. Brockleshy, D. Monroc, A. F. J. Levi, M. Kong, S. I Licu, J Kwo, C. E. Rice, P. M. Mankiewich, and R. E. Howard, Appl. Phys Let1. 54, 1175 (1989).

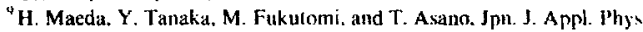
27, L209 (1988).

${ }^{10}$ C. N. R. Rao. L. Gamapathi. R. Vijayaraghavan, G. Ranga Rais. K Murphy, and R. A. Mohan Ram, Physica C 156, 827 (1988).

"M. A. Subramanian, C. C. Torardi. J. C. Calabrese, J. Gopalakrishnan. K. J. Morrissey, T. R. Askew, R. H. Flipen, U. Chowdhry, and A. W. Sleight. Science 239. 1015 (1988) 